Revista Calidad en la Educación Superior

Programa de Autoevaluación Académica

Universidad Estatal a Distancia

ISSN 1659-4703

Costa Rica

revistacalidad@uned.ac.cr

\title{
EDUCACIÓN SUPERIOR EN CENTROAMÉRICA.
}

\author{
HIGHER EDUCATION IN CENTRAL AMERICA
}

\author{
Roberto de J. Mora Sánchez ${ }^{1}$ \\ Roberto.mora.sanchez@una.cr \\ Universidad Nacional de Costa Rica
}

\author{
VII Edición \\ Volumen 4, Número 1 \\ Mayo 2013 \\ pp. $187-198$
}

Recibido: noviembre, 2012

Aprobado: abril, 2013

\footnotetext{
${ }^{1}$ Máster en Administración Educativa, administrador de empresas y contador privado. Trabajó como Ejecutivo Institucional del Colegio Científico de Costa Rica en la Universidad Nacional de Costa Rica Sede Región Brunca. El Consejo Iberoamericano le otorgó en Honor a la Calidad Educativa y la Honorable Academia Mundial de Educación en el año 2010 el VII Premio Iberoamericano a la Excelencia Educativa. Master en Gestión Pedagógica Iberoamericana y un Doctorado Honoris Causa de Iberoamérica. Académico de la Universidad Nacional de Costa
} 


\section{Resumen}

El presente artículo reseña el origen de la educación superior centroamericana desde sus comienzos hasta nuestros días. Se hace un breve recorrido por el origen de la educación superior, su devenir en América Latina y hasta el origen de la primera universidad centroamericana. Se exponen brevemente aquellos sucesos de orden político, económico o social de la época que influyeron en el surgimiento de la educación superior estatal en cada país de la región, así como los que determinaron el origen de la educación superior privada con sus promotores y detractores. Se expone el caso guatemalteco, hondureño, salvadoreño y nicaragüense y su evolución a nuestros días, así como los esfuerzos que han realizado los países de la región en procura de mejorar la calidad de la educación terciara que ofrecen las universidades públicas y privadas aquí establecidas.

Palabras clave: Educación Superior, Educación Superior Pública, Educación Superior Privada, Universidades Públicas, Universidades Privadas, Origen de la Educación Superior, Centroamérica.

\section{Abstract}

This article reviews the origins of American higher education since its inception to the present day. A brief tour of the origin of higher education, its evolution in Latin America and even the origin of the first American university. It outlines those events of a political, economic or social of the time that influenced the emergence of state higher education in each country of the region, as well as those for which the origin of private higher education with its proponents and detractors. We present the case of Guatemala, Honduras, El Salvador and Nicaragua and its evolution to the present day, as well as the efforts made by the countries of the region in an effort to improve the quality of tertiary education offered by public and private universities established herein.

Keywords: Higher Education, Public Higher Education, Private Higher Education, Public Universities, Private Universities, Origin of Higher Education, Central America.

\section{Introducción}

Existen diversos artículos que exponen el origen de la educación superior, o el origen de la educación superior latinoamericana, pero poco se ha escrito sobre el origen de la educación superior en Centroamérica. De ahí la importancia de este artículo que resume parte de la historia de la evolución de la educación superior, 
su paso por América Latina, pero más específicamente en Centroamérica y su situación en los países de Guatemala, Honduras, El Salvador y Nicaragua.

La educación superior denominada universidadse origina en el siglo XII. El término utilizado para este tipo de organización originalmente era universitas, palabra que se utilizaba para designar según Cobban (1975) "cualquier agregado o cuerpo de personas con intereses comunes y un estatuto legal independiente, sea un gremio o una corporación municipal" (p.23). En ese sentido el uso del término aplicaba para un grupo de profesorado y estudiantes cuyo interés era promover el conocimiento, la investigación y la acción social a través del aprendizaje de ciertas destrezas vocacionales para favorecer las necesidades del mercado, la iglesia y la sociedad.

Únicamente las personas con licencia para enseñar dentro del gremio universitario eran quienes estaban autorizados para formar a los profesionales de la sociedad. Algunas especialidades de la época según Bravo y Guzmán (1998) eran: "teología, derecho, retórica, o las artes liberales y las ciencias quese estimaban apropiadas para el entrenamiento del clero profesional, de losabogados y administradores eclesiásticos y civiles" (p.23).

Rama(2006) afirma:

América Latina inició durante el Siglo XX, al calor de las banderas de la Reforma de Córdoba de 1918, un modelo específico de Universidad caracterizado por la autonomía de sus instituciones públicas, un marco de gestión basado en la modalidad de cogobierno, la presencia destacada de la educación monopólica pública, la gratuidad en su acceso, una estructura corporativista de gestión a su interior, y un rol del estado mayoritariamente orientado al suministro de los recursos financieros. Este modelo agregaba entre otros elementos distintivos una excesiva rigidez de los marcos jurídicos; la ausencia de mecanismos institucionalizados de evaluación, una estructura institucional basada en facultades y un sistema de gestión donde lo legislativo, lo ejecutivo y lo judicial se integraba en los Consejos Universitarios (p.1). 
A Centroamérica la universidad llegó con motivo de la conquista europeaespañola, junto con el concepto de la iglesia gracias a una imposición de las órdenes reales españolas y religiosas a favor de la educación de las tierras nuevas conquistadas. Éstas iniciaron en México, país fronterizo de la región centroamericana y según Brunner (1990) "desde 1538 y 1827 se crearon en la América Hispana un total de 33 universidades, algunas de las cuales tuvieron una existencia puramente nominal" (p.5); sin embargo la primer universidad Centroamericana fue la Universidad San Carlos Borromeo, de Guatemala, extendiéndose hasta el año 1841, año en que se funda la Universidad de El Salvador. Fue la Universidad San Carlos la responsable de la formación exclusiva de profesionales centroamericanos durante alrededor de 160 años desde el año 1841 según el Ministerio de Educación de El Salvador, año en que se acepta que fue creada la Universidad de El Salvador vía decreto con énfasis en derecho, matemática, la gramática castellana y la docencia libre.

Es hasta el año 1972 que se crea la Ley Orgánica de la Universidad de El Salvador, en 1965 la Ley de Universidades Privadas de El Salvador y hasta 1995 que se crea la Ley de Educación Superior de El Salvador. (Rodríguez T., 2003, p.2.).

En la Universidad de San Carlos de Guatemala, que nace en el siglo XIX, ...la otorgación de grados académicos cae a sólo un tercio del número otorgado en el decenio anterior. (Brunner, 1990 p. 7).

El surgimiento de estas universidades es gracias a la complicidad del gobierno de estos países porque quienes los presiden conciben su establecimiento como una prolongación del Estado y tenían las expectativas de mantener una estrecha relación con sus autoridades académicas. Algunas de estas universidades operan bajo la tutela del Ministerio de Educación y otras con un concejo de educación superior estatal. 
Ya en 1940 existían en Centroamérica un número de universidades estatales y para entonces se había iniciado el proceso de diferenciación de la enseñanza superior.

Es menester indicar que el ritmo de proliferación de las universidades estatales había sido muy lento durante el siglo XIX, apenas comienza a tener síntomas de apuro a inicios del siglo XX en Centroamérica. La siguiente tabla muestra la matrícula en Educación Superior y tasas brutas de escolarización en Centroamérica durante 1950:

Se observa en la tabla No. 1 la marcada diferencia en la tasa bruta de escolarización de los países de Panamá y Costa Rica en relación con el resto de los países centroamericanos, cuya tasa oscilaba entre 0.6 y 0.8 por cada cien mil habitantes.

Panamá para 1960 había apostado a la educación y para ese año alrededor del $15 \%$ de sus graduados eran de las facultades de ciencias médicas, mientras que para el resto de Centroamérica únicamente se lograba el 10\%.

Tabla No. 1

Centroamérica: matrícula en la Educación Superior y tasas brutas de escolarización superior alrededor de 1950

\begin{tabular}{lrr}
\hline \multicolumn{1}{c}{ País } & $\begin{array}{c}\text { Matrícula en } \\
\text { Educación } \\
\text { Superior }\end{array}$ & $\begin{array}{c}\text { Tasa bruta de } \\
\text { escolarización } \\
\text { superior }\end{array}$ \\
\hline Guatemala & 2373 & 0.8 \\
El Salvador & 1199 & 0.6 \\
Honduras & 818 & 0.6 \\
Nicaragua & 560 & 0.6 \\
Costa Rica & 1539 & 2.0 \\
Panamá & 1519 & 2.2 \\
\hline
\end{tabular}

Fuente: adaptado de Brunner, (1990) 
Fue después del año $1960^{2}$ que el Estado comienza a desatender financieramente a las universidades debido a que no podían sostener la tasa de crecimiento de las matrículas, además el sector público en varios países latinoamericanos y centroamericanos tuvo que restringir las admisiones de los nuevos estudiantes a la educación superior mediante pruebas de ingreso o admisión, debido a que el crecimiento desmedido de estudiantes en las universidades estatales generaba baja calidad en la formación de profesionales. (Knut, 1999, p. 22).

Mientras el Estado se detiene en las discusiones presupuestales, surge la universidad privada como una respuesta al modelo tradicional y con más fuerza entre los años ochenta y noventa con la finalidad de atender la demanda en la educación superior para una clase trabajadora, media y alta que podía sostener los costos de manutención en educación superior privada. El incremento de la educación superior privada en los últimos treinta años ha rondado a más del 1\% por año y ha alcanzado más del $70 \%$ de cobertura en toda la región centroamericana. Es así como la educación superior viene a reformarse pasando de lo ideológico, político y autónomo a lo mercantil y lo privado, donde privan las necesidades del mercado sobre la ideología del estado. (Knut, 1999, p. 22).

Es entonces en adelante que aparecen dos escenarios en educación superior, el escenario regulado por la autonomía universitaria y el estado y el escenario regulado por las demandas sociales y mercantiles en la educación superior, es decir lo estatal y lo privado.

\footnotetext{
2 Hasta 1960 todas las universidades en Centroamérica eran públicas, de tal manera que las discusiones y polémicas sobre su funcionamiento tenían que ver con la autonomía, los presupuestos y las elecciones de sus autoridades. Hasta 1960, aproximadamente, las universidades recibían a relativamente pocos estudiantes, ya que la masificación de la educación primaria y secundaria todavía no producía suficientes bachilleres como para abarrotar las aulas universitarias. Sin embargo, a partir de la década de 1960, la educación universitaria adquirió un nuevo atractivo, especialmente entre las capas medias que comenzaban a demandar más educación para sus hijos. No debe sorprender, entonces, que durante esta década se incrementó notablemente la matrícula universitaria y se fundaron las primeras universidades privadas en la región. Knut Walter, (1999, p. 22)
} 
Ante este nuevo paradigma se han incrementado y diversificado las instituciones de enseñanza superior privada en toda la región centroamericana como respuesta a dos ejes sociales de presión, la cantidad de graduandos de los centros de enseñanza media y las necesidades insatisfechas en los mercados por parte de los centros de enseñanza superior estatal, tema que ha llamado altamente la atención de la sociedad civil que desea profesionalizarse debido a los reducidos años de formación para licenciarse y la liberación de los requisitos de ingreso en la mayoría de las universidades privadas.

En 1994 según Rama(2006), "la región ya tenía 5.438 instituciones de educación superior, de las cuales 2.515 eran públicas y el 53,71\% eran privadas.", (p.8). En cuanto a matrícula en educación superior, a fines de siglo ésta ya superaba el $50 \%$ en las universidades privadas.

En el caso hondureño según Duriez(2011) por ejemplo, "Para el año al año 2010 existen 20 universidades, de las cuales 6 son públicas y 14 privadas. La Universidad Nacional Autónoma de Honduras recibe el 6\% del presupuesto de ingresos netos de la República y las demás universidades estatales reciben presupuesto pero en menor proporción..."

Al 2006 la UNAH no contaba con un sistema de admisión a la Educación Superior basado en Pruebas de Aptitud Académica... La cobertura de la Educación Superior al 2009 llegó al 14,5\% de la población estimada entre 18 y 24 años y la tasa promedio anual de matrícula fue del $3,3 \%$ entre el 2005 y 2009 ... Mientras la tasa promedio anual de matrícula en educación superior estatal disminuyó -1,6\%, creció al $17,6 \%$ en la privada al año $2009 . .$. En el año 2010 las universidades privadas tienen mayor representación atendiendo a un 38\% de la población estudiantil y las públicas el $62 \%$ restante. (p. 5).

En el caso de El Salvador según Hasbún (2007), 
En la actualidad existen en el país 25 universidades privadas autorizadas por el Ministerio de Educación, incluida la Leonardo Da Vinci, además de la estatal Universidad de EI Salvador (UES).La UES, fundada el 16 de febrero de 1841, era la única universidad en el país hasta 1965, cuando se aprobó la Ley de Universidades Privadas que dio paso a la fundación, en septiembre de ese año, de la Universidad Centroamericana "José Simeón Cañas" (UCA), administrada por jesuitas. En años posteriores y hasta 1978 fueron fundadas otras tres universidades privadas. Sin embargo, la proliferación de esos centros de estudios ocurrió a partir de 1981, luego de la intervención militar de la universidad nacional, en junio de 1980, que duró más de dos años, en los inicios de la guerra civil que vivió el país (19801992).La UES era considerada por las autoridades como un "nido de guerrilleros" y el Ministerio de Educación concedió grandes facilidades para la apertura de universidades privadas, al grado que hacia 1990 existían 42; muchas instaladas en pequeños espacios y con dudosa calidad académica. La Ley de Educación Superior fue aprobada en septiembre de 1995 y estableció un plazo de dos años para que las universidades se adecuaran a las exigencias de la misma.

En el caso nicaragüense según Cisneros (2004), "Nicaragua no tiene un Sistema de Educación Superior estructurado como tal", (p.2). Es un subsistema perteneciente al Ministerio de Educación, por ejemplo la tasa de escolaridad terciaria en Nicaragua al año 2000 indicaba que por cada 6,67 personas que ingresaban a la educación superior, se egresaban 2.46 , debido a que los niveles de conocimientos básicos en español y matemática son muy bajos en el país y es el país con más bajas tasas de escolaridad; por ejemplo en el año 2000 mientras Costa Rica tenía una tasa de escolaridad terciaria del 35\%, Nicaragua tenía el $12 \%$ al igual que Honduras y sólo superando a Guatemala que estaba en un $10 \%$. Era hasta el año 2005 que se esperaba en Nicaragua contar con un Sistema Nacional de Acreditación Universitaria con 33 universidades que iniciaron en el año 2000 procesos de autoevaluación institucional.

Según Cisneros (2004), "Al año 2004 se cuenta con un total de 46 instituciones universitarias en Nicaragua, las que se desglosan de la siguiente manera, 4 estatales pertenecientes al CNU (Consejo Nicaragüense de Universidades), 6 privadas pertenecientes al $\mathrm{CNU}, 2$ estatales no pertenecientes al $\mathrm{CNU}, 32$ 
privadas autorizadas por el CNU, 2 privadas extranjeras. La población estudiantil estimada al año 2004 es de 105 mil estudiantes". (p. 8).

Al reflexionar sobre los aportes de Rama, Duriez, Hasbún y Cisneros, el fenómeno del crecimiento de la educación superior privada llegó a nuestra región sin fines de eclipsarse, sino más bien su proliferación ha venido en aumento. Es por ello que autoridades académicas, gobernantes y pensadores centroamericanos han optado por la creación de un consejo que permita certificar la calidad de los diferentes centros de educación superior en la región.

Después de 1990 varios países de la región han aunado esfuerzos para crear el Consejo Superior de Universitario Centroamericano (CSUCA). Este consejo se creó con el propósito de establecer mecanismos regionales que armonicen, articulen e integren el esfuerzo de diversas instituciones y organizaciones en el ámbito centroamericano, y den validez internacional a la acreditación de la calidad de la educación superior que se realiza en los distintos países de la región. (http://www.cca.ucr.ac.cr/acerca_de/historia).

Para su creación, tanto los Ministerios de Educación de América Central, como el Consejo Superior Universitario Centroamericano (CSUCA) coordinaron esfuerzos para la creación de este organismo.

Desde el año 2000, el CCA cuenta con un Sistema Interno de Aseguramiento de la Calidad (SIAC), como mecanismo para garantizar la calidad de su quehacer y gestión y en respuesta a la necesidad de que al igual que las instituciones de educación superior, las agencias de acreditación, y en este caso el CCA como agencia de segundo nivel, implementen mecanismos de aseguramiento y mejoramiento continuo de su calidad.

La tabla No. 2 muestra las instancias de cada país centroamericano y el estado actual en acreditación universitaria pública y privada ante el CCA: 
Tabla No. 2

Consejo Centroamericano de Acreditación

Países según instancia y estado de acreditaciones, al 18 de marzo de 2013

PAís

INSTANCIA

ESTADO DE LAS ACREDITACIONES

\begin{tabular}{|c|c|c|}
\hline Guatemala & Sin datos & $\begin{array}{c}\text { Universidad San Carlos } \\
\text { con dos carreras de } \\
\text { ingeniería }\end{array}$ \\
\hline Belice & Sin datos & Sin datos \\
\hline Honduras & Sin datos & \\
\hline EI Salvador & $\mathrm{CdA}$ & $\begin{array}{l}\text { Autorizados para } \\
\text { acreditaciones }\end{array}$ \\
\hline Nicaragua & CNEA & $\begin{array}{l}\text { No han iniciado } \\
\text { acreditaciones. }\end{array}$ \\
\hline Costa Rica & SINAES & $\begin{array}{l}\text { Autorizados para } \\
\text { acreditaciones }\end{array}$ \\
\hline Panamá & CONEAUPA & $\begin{array}{l}\text { No han iniciado } \\
\text { acreditaciones solo la } \\
\text { Universidad de Panamá } \\
\text { con una carrera de } \\
\text { ingeniería. }\end{array}$ \\
\hline
\end{tabular}

Fuente: Adaptado de http://www.cca.ucr.ac.cr/node/49

Es necesario impulsar la integración de la región en el programa de acreditaciones del CCA porque esto favorece el compromiso de cada país con mejoramiento de la calidad de su enseñanza universitaria, ya sea pública o privada.

No obstante, debe existir una política nacional impulsada por los gobernantes de cada país centroamericano y el mismo CCA para garantizar no solo calidad, sino también democracia al acceso de los servicios que ofrecen educación superior estatal o privada.

\section{Conclusiones}

El surgimiento de las universidades en el siglo XII y su incidencia durante el período de la conquista española a los países centroamericanos constituye la coyuntura ideal para su establecimiento en la región. 
El Estado concibe necesario y oportuno establecer universidades bajo su tutela, financiamiento y supervisión como una oportunidad para ampliar su cobertura y dominio social con la expectativa de la complicidad de las autoridades académicas antes de 1940 en la región.

Después de 1960 las universidades centroamericanas estatales son presa del crecimiento demográfico, la demanda insatisfecha de la ciudadanía, la desatención financiera gubernamental y comienzan a restringir la admisión por medio de pruebas de aptitud o mecanismos que les permitieran filtrar a los estudiantes.

De 1960 en adelante surgen las universidades privadas centroamericanas como un mecanismo para atender la demanda insatisfecha de educación terciaria en la región y como respuesta a la desatención estatal a la educación superior pública centroamericana.

Después de 1990 ha existido una gran preocupación en todos los países centroamericanos por el mejoramiento de la calidad en educación superior. Es por ello que se crea el Consejo Superior de Universitario Centroamericano (CSUCA) como respuesta a la necesidad de asegurar la calidad de los servicios de educación superior pública y privada en toda la región.

\section{Bibliografía}

Bravo, A. y Guzman, A. (1998). Hacia un proyecto de Universidad alternativa. Sociedad de NicolaitasExAlumnos, A.C.Universidad Michoacana de San Nicolás de HidalgoQUEHACER NICOLAITA. México.

Brunner, J. J.(1990).Educación Superior en América Latina, Cambios y Desafíos. Editorial Fondo de Cultura Económica, Chile.

Cisneros, E. (2004). Características Generales de la Educación Superior en Nicaragua. Recuperado de http://tuning.unideusto.org/tuningal/images/stories/presentaciones/nicaragua _doc.pdf 
Cobban, A.B. (1975). The Medieval Universities: Their Development and Organization, Methuen.Londres, pág. 23.

Duriez M. (2011). La educación superior Iberoamérica 2011, la educación superior en Honduras 2005 - 2009.

Hasbún, C. (2007). Inminente cierre universidad privada en El Salvador afecta a 276 estudiantes. Terra Networks México S.A. Recuperado de http://www.terra.com.mx/articulo.aspx?articuloid=317116

Knut, W. (1999). La educación en Centroamérica:Reflexiones en torno a sus problemas y su Potencial. Recuperado de http://ca2020.fiu.edu/Themes/Knut_Walter/Walter.pdf

Rama, C. (2006).La Tercera Reforma de la educación superior en América Latina.Fondo de la Cultura Económica, Buenos Aires, Argentina.

Rodríguez, A. (2003). El Sistema de Educación Superior en El Salvador. Dirección Nacional de Educación Superior. Recuperado de http://tuning.unideusto.org/tuningal/images/stories/presentaciones/el_salvad or_doc.pdf

Universidad de Costa Rica (2013). Recuperado dehttp://www.cca.ucr.ac.cr/acerca_de/historia 